III.

\title{
Anatomische Untersuchungen über Heterochromie bei tauben, unvollkommen albinotischen Katzen.
}

\author{
Von \\ Dr. HANS LAUBER, \\ Assistenten der I. Augentlinik in Wien.
}

Dic Frage des partiellen Albinismus und seiner Bedeutung als Entwicklungsanomalie hat in der letzten Zeit an Bedeutung gewonnen. Es scheint die Funktion des Sehorganes an die Anwesenheit von Pigment gebunden zu sein. Sehen wir ja, dass die Ocellen und Ommen der wirbellosen Tiere und die Augen der Wirbeltiere als einen wesentlichen Teil des physiologisch wichtigen Endapparates pigmentierte Zellen besitzen. Auch im Gehörorgane ist die Anwesenheit pigmentierter Zellen eine der charaliteristischen Eigenschaften des Organes [Alexander (2)]. Es hat laher die früher bekannte Tatsache, dass völlig oder teilweise alhinotische Tiere häufig taub sind, durch die Kenntnis der vergleichend anatomischen und physiologischen Tatsachen an Bedeutung \%ugenommen.

Abelsdorff (1) hat die Augen eines blaüugigen tauben Hundes untersucht und berichtet, lass ausser den Fehlen des Pigmentes in der gesamten $\mathrm{U}$ yea keine von der Norm abweichenden pathologischen Zustände im Auge nachweisbar waren.

Ich habe Gelegenlseit gehabt, die Augen zweier tauber weisser Katzen, die je ein braungelbes und ein blatues d uge hatten, anatomisch zu untersuchen. An beiden Tieren war ausser der Taubheit nichts Ungewöhnliches zu bemerken. I)as eine Tier habe ich selbst beobaclitet, das andere Dr. Alexander, der mir die Augen überliess und selbst das Gehörorgan untersuchte $(3,4,5)$. Beide l'iere habe ich in viro nicht ophthalmoskopiert, da ich die Bedeutung der Heterochromie zurzeit nicht würdigte.

Während der anatomische Befund am braunen Auge vollständig mit dem anderer Augen, die normalen Tieren entstammten, übereinstimmt, ist dies bei den beiden blauen Augen, die einander in allen Beziehungen vollständig gleichen, nicht der Fall.

Da die Abweichungen von der Norm ausschliesslich die Pigmentierung betreffen, schicke ich die Beschreibung der pigmentierten Elemente der normalen Katzenaugen voraus.

Im normalen Auge der Katze haben wir vier verschiedene Arten von pigmentierten Zellen $z u$ unterscheiden: 
1. Die Zellen des Pigmentepithels der Retina, die polygonal, meist regelmässig sechseckig sind und ein dunkelbraunes, etwas grünliches Pigment in der Form von Fuscinnadeln enthalten. Vor der Ora serrata machen die Fuscinnadeln einer grobkörnigen Form des Pigmentes Platz, das sich im retinalen Pigmentepithel des vorderen Bulbusabschnittes findet. Das Pigment liegt vorwiegend in der Peripherie der Zellen, so dass der runde, zentral gelegene Kern deutlich hervortritt. An der Rückfliche der Iris erfïhrt dies Epithel die bekannte Umwandlung in den Dilatator iridis, wobei jedoch das Pigment morphologisch dem des Ciliarkörpers gleichbleibt. Da diese Zellen ein ununterbrochenes Ganzes bilden, fasse ich sie in eine Gruppe zusammen. Ebenso gehören hierher die aus der inneren Wand der sec. Augenblase hervorgegangenen Epithelzellen der Irishinterfläche, die ein morphologisch gleiches Pigment enthalten.

2. Die sogenannten Klumpenzellen der Iris, deren Al)stammung rom retinalen Pigmentepithel E ls chnig und Verfasser (6) beim Menschen nachgewiesen haben. Sie sind von unregelmässiger Form, sehr dicht pigmentiert. Morphologisch ist ihr Pigment dem retinalen Pigmentepithel gleich. Der Kern ist in nicht entpigmentierten Prïparaten nur schwer sichtbar, da er fast völlig von Pigment verdeckt wird.

3. Die pigmentierten Zellen in der Chorioidea und Sklera. Sie sind von sehr unregelmäissiger Gestalt, gewöhnlich mit mehreren Fortsiitzen versehen. Das Pigment, das den zentral gelegenen, anselınlichen Kern fast stets leicht erkennen lässt, besteht aus ziemlich grossen, rundlichen Körnern, niemals aus Kristallen oder Nadeln, und ist von dunkelbrauner Farbe, jedoch heller als das Pigment der früheren beiden Zellarten. Die in der Sklera vorkommenden Zellen sind gewöhnlich stärker pigmentiert, auch ist ilır Pigment hïufig grobkörniger. Diese Zellen stellen in der Chorioidea die einzigen pigmentierten Elemente dar; ihre Anzahl nimmt in Ciliarkörper bedeutend ab. In der Pars scleralis corporis ciliaris, besonders in der unmittelbaren Nilue der Sklera, sind sie zahlreicher, im Balkenwerk des Ligamentum pectinatun sind sie spärlich. Im hinteren Abschnitte der Pars iridica corporis ciliaris sind sie spärlich und kommen in der Iriswurzel nur vereinzelt vor. In der Iris selbst fehlen sie vollstïndig.

4. Die Pigmentzellen der Iris und des Ciliarkörpers. Es sind dies grosse, spindelförmige Zellen mit meist ovalem, mit Hämatoxylin deutlich färbbarem Kerne, meist mit sehr schlanken Fortsïtzen versehen. Sie sind von einem hell gelbbraunen, iuusserst feinkörnigen l'igment erfüllt; es ist so fein, dass sich die einzelnen Körner sogar mit der lmmersion nur schwer unterscheiden lassen. Aus ihnen besteht zum grössten Teil die Iris, wobei sie vorwiegend in einer zur Oberfliche senkrechten Richtung liegen; von dieser Richtung weichen sie ab, um grössere Gefässe mit einem Mantel zu umgeben. An der Irisoberfläche liegen sie parallel zu 
ihr und sind an dieser Stelle am dichtesten pigmentiert. Sie kommen auch in der Pars iridica corporis ciliaris und im ligamentum pectinatum vor.

Die Zellen der ersten beiden Gruppen sind ektodermaler Abstammung, indem sie alle aus den Zellen der sec. Augenblase hervorgehen. Die Zellen der beiden letzten Gruppen sind mesodermaler Abstammung.

Während nun die Zellen ektodermaler Abstammung in den blauen A ugen vollständig den korrespondierenden Zellen der braunen Augen gleichen und in ihrer Pigmentierung keine $A b w e i c h u n g$ erkennen lassen, fehlt das Pigment in den Zellen mesodermaler Abkunft vollständig. Die Zellen selbst sind gut erkennbar, anscheinend gleich gebaut wie die der braunen Augen. Es fehlt also das Pigment, sowohl das dunkelbraune der Chorioideal- und Skleralzellen, als auch das hellbraune der Iriszellen.

Wie mir Herr Dr. Alexander mitteilt, fehlt an den Gehörorganen der einen Katze, die er untersucht hat, wie in allen ähnlichen Fällen, das Pigment in den Zellen des perilymphatischen Bindegewebes, das normalerweise Pigmentzellen enthält, die den chorioidealen Pigmentzellen vollkommen entsprechen. Dieser Befund muss nach den Untersuchungen von Abelsdorff, Alexander und Tandler als typisch für die tauben, unvollkommen oder vollkommen albinotischen Katzen und Hunde gelten. Gleich\%eitig bestanden Missbildungen der knöchernen und membranösen Schnecke.

Diese Befunde stimmen vollständig mit denen Abelsdorffs überein. Nur erwähnt Abelsdorff die normalerweise auch beim Hunde vorkommenden Klumpenzellen der Iris gar nicht. In meinen beiden Fällen waren sie sehr deutlich vorhanden. Ihr Fehlen in der Iris des von Abelsdorff untersuchten Hundes wäre nur dann verständlich, wenn man annehmen würde, solche versprengte Zellen epithelialer Abstammung seien bei dem Individuum überhaupt nicht dagewesen.

Aus den angeführten Tatsachen lässt sich der Schluss ziehen, disss die Pigmentbildung nicht bloss der neuroepithelialen Bestandteile der höheren Sinnesorgane, sondern auch deren bindegewebiger Hüllen in einer gewissen Beziehung zu ihrer Funktion steht. Das Fehlen des Pigmentes ist als ein Zeichen der Minderwertigkeit der Organe aufzufassen, wie denn überhaupt der vollkommene oder unvollkommene Albinismus als eine Art Missbildung, die Albinos als physisch minderwertige Individuen erscheinen. Ein Beweis dafür ist die physiologische Minderwertigkeit albinotischer Augen beim Menschen, die eine längst bekannte Tatsache ist. Mit Rücksicht auf die in letzter Zeit von Fuchs ( 7 ) und Weill (8) vertretene Ansicht, dass die mangelhafte Pigmentierung eines Auges als Krankheitsursache oder wenigstens als zur Krankbeit prädisponierendes Moment zu betrachten sei, muss die Tatsache besonders betont werden, duss sich pathologische, insbesondere entzündliche Verïnderungen irgend welcher Art in den unter- 
suchten blauen Augen nicht finden liessen. Das beweist wohl, dass Pigmentmangel an sich keine Krankheit oder Krankheitsursache ist. Die beschriebenen Fälle stellen lediglich eine anatomische Variante dar, indem sie eine Mittelstellung zwischen den normal gebildeten blauen Augen darstellen, deren Iris frei von mesodermalem Pigment ist, deren Chorioidea dagegen pigmentiert ist, - und den vollständig albinotischen Augen, denen mesodermales sowohl als auch ektodermales Pigment fehlt.

Dass eine helle Fürbung, besser gesagt, eine Entfärbung der Iris, krankhaft sein kann, steht unbestreitbar fest. Sie kommt als Folge akuter oder chronischer Entzündung beiderseitig oder auch einseitig vor. In Fällen chronischer Iritis entstehen weitgehende Veränderungen im Auge, die sich klinisch als Präzipitate auf der hinteren Hornhautoberfäche, träge Pupillarreaktion, Defekte im pupillaren Pigmentsaume der Iris, Verlorengehen der Struktur der Irisoberfläche, die zur Glätte führt, Verdünnung der Iris bis zur Durchleuchtbarkeit einzelner Stellen des Pupillaranteiles, Entfärbung der Iris, Kataraktbildung, Entstehung von Glaskörperfüden kenntlich machen. Was insbesondere die Veränderungen der Iris betrifft, so kann die helle Farbe bei dem äusserst chronischen Verlaufe und dem Fehlen subjektiver Symptome als erstes Anzeichen der Krankheit sowohl dem Patienten als auch dem Arzte auffallen. Die Farbe solcher Irides gleicht auch nicht derjenigen normaler blauer oder grauer Augen und wäre wohl am besten mit "fahl" bezeichnet. Der anatomische, von Fuchs erhobene Befund spricht meines Erachtens auch für eine äusserst chronische Entzündung; das Ergebnis ist das $\mathrm{Zu}$ grundegehen der pigmentierten Stromazellen und die Bildung von Bindegewebe. Das Fehlen der pigmentierten Stromazellen dürfte wohl als Folge der Erkrankung zu betrachten sein, nicht als kongenitale Veränderung. In diesem Sinne spricht sich auch Fuchs selbst aus.

Aus den angeführten Tatsachen lässt sich sonach folgender Schluss ziehen: Die Verschiedenfarbigkeit der Iris (Heterochromie) kann entweder eine angeborene Anomalie sein, als Ausdruck partiellen Albinismus, wie in den beiden beschriebenen Fällen; oder sie ist eine krankhafte Veränderung, die als Folge, nicht als Ursache einer Erkrankung anftritt und hesonders dann auffällt, wenn die Krankheit einseitig und nicht, wie so hüufi, doppelseitig ist.

\section{Literatur.}

1. Abe!sdorff, G., Ueber Blauäugigkeit und Heterophthalmus bei taubec albinotischen lieren. Arch. f. Ophthalmologie. LIX. p. 376. 1904.

2. Alexander, G. Weitere Studien am Gebörorgane unrollkommen albino. tischer weisser Katzen. Arch. f. Ohrenbeilkunde. LI.

3. Derselbe, Zur vergleichenden pathologischen Anatomie des Gehörorganes. I. Gehörorgan und Gehirn einer unvollkommen albinotischen weissen Katze. Arch. f. Obrenheilkunde. LI.

4. Derselbe, Weitere Studien am Gebörorgane unvollkommen albinotischer Kat\%en. Zeitschr. f. Ohrenheilkunde. XIVIII. 
5. Alexander und $\mathrm{T}$ andler, Untersuchungen an kongenital tauben Hunden, Katzen und an jungen kongenital tauben Katzen. Arch. f. Obrenbeilkunde LXVI.

6. Elschnig, A., Ueber die sogenannten Klumpeuzellen der Jris. Sitzungsbericlit d. K. K. Akademie der Wissenschaften. Wien. 1906. p. 174.

7. Fuchs, E, Ueber Komplikationen der Heterochromie. Zeitschr. f. Angenheilkunde. XV. p. 191. 1906.

8. Weill, G., Ueber Heterophthalmus. Zeitschr. f. Augenheilkunde. XX. p. 165. 1904

\section{IV.}

Aus der Kgl. Universitäts-Augenklinik \%u Berlin.

(Direktor Geb. Rat Prof. v. Jichel.)

\section{Hyperopie und Diabetes mellitus.}

Vou

\section{Dr. ERNST LICHTENSTEIN.}

Unter diesem Titel berichtet Gallus in Aprilheft dieser Zeitschrift von einen Patienten mit diabetischer Hyperopie, den er in seiner Praxis zu untersuihen Gelegenheit hatte. Er stellt in seiner Arbeit auch die bisher mitgeteilten fünf anderen Fälle dieser Art zusammen und gibt die wesentlichsten Punkte ihrer Krankengeschichte wieder. Gemeinsam ist allen sechs Fällen, dass sie erst nach Entstehung der Hyperopie zur Beobachtung kamen und somit exakte Untersuchungsresultate aus der Zeit vorher nicht vorhanden sind. Aus diesem Mangel erklärt es sich wohl, dass eine Reihe ron Autoren, wie 7. B. SchmidtRimpler und Groenow, überhaupt noch nicht an eine diabetische Hyperopie glauben wollen, sondern in den bisher publiøierten Fällen dieser Art an die Manifestation einer bisher latenten Hypermetropie denken.

Ich walr nun in der Isage, einen Patienten, bei dem später eine diabetische transitorische Hyperopie auftrat, schon in der Zeit vor ihrem Entstehen z.u untersuchen und dann längere Zeit in Beobachtung \%u behalten. Es sei mir daher gestattet, in Kïrze ron diesen Falle zu berichten, der meines Erachtens einwandsfrei zeigt, dass es eine diabetische transitorischeHyperopie wirklich gibt.

Der 17jährige Gärtner T. H. kommt am 8. III. 1906 in die Kógl. Universitäts-Augenklinik mit der Klage, class er seit einigen 'Tagen nicht mehr gut sehen kōnne. Die Augenuntersuchung ergibt folgendes liesultat:

Geringe Anisokorie. I. $>$ R. Pupillarreaktion beiderseits prompt. Brechende Mledien klar. Fundus oline Besonderheiten. Gesichtsfeld beiderseits auch für l'arben völlig normal.

Funktionell: Beiderseits konvex 1,j Dioptr., S. $={ }^{6} / 6$. Skiaskopisch ehenfalls beiderseits konvex 1,j Dioptr. Dagegen braucht der Patient, um in der 